\title{
Analysis of nonhydrostatic high-pressure diffraction data (cubic system): Assessment of various assumptions in the theory
}

\author{
Anil K. Singh ${ }^{\text {a) }}$ \\ Materials Science Division, National Aerospace Laboratories, Bangalore 560017, India
}

(Received 23 February 2009; accepted 10 July 2009; published online 25 August 2009)

\begin{abstract}
The mathematical formulation commonly used to analyze the high-pressure diffraction data from the sample under nonhydrostatic compression is based on three assumptions: A1—a weighted harmonic mean of the diffraction shear moduli under Reuss and Voigt limits with a weight parameter $\alpha$ that lies between 0.5 and 1 describes adequately the diffraction shear modulus; A2 - a stress tensor with only the diagonal terms describes the stress state at the center of the sample under nonhydrostatic compression; and A3 - the lattice-strain equations derived using only the linear elasticity theory are adequate to derive strength and elastic moduli from the diffraction data. To examine A1 we derive compressive strength, diffraction shear moduli, and single-crystal elastic moduli from the experimental high-pressure x-ray diffraction data on bcc $\mathrm{Fe}, \mathrm{Au}, \mathrm{Mo}$, and $\mathrm{FeO}$. These data contain plastic deformation effects. The diffraction shear modulus in the limit of small deformation (elastic) is computed using rigorous formulae derived by Kröner [Z. Phys. 151, 504 (1958)] and de Wit [J. Appl. Crystallogr. 30, 510 (1997)]. The elastic moduli are derived from the computed shear moduli assuming the validity of A1. The results show that A1 with $\alpha \cong 0.5$ is valid for small deformation in all four cases. The analysis of the experimental data suggests that $\mathrm{A} 1$ is valid with $\alpha<1$ for solids with $x>1$ where $x=2 C_{44} /\left(C_{11}-C_{12}\right)$; for solids with $x<1$, the validity of A1 requires $\alpha>1$. At least for solids of the cubic system, the effect of plastic deformation appears to be fully contained in a single parameter $\alpha$. In practice, deviations from A2 of varying magnitudes occur mainly because of the difficulty in avoiding diffraction from regions of stress gradient in the sample. A discussion of A3 is presented. () 2009 American Institute of Physics.
\end{abstract}

[DOI: $10.1063 / 1.3197213$ ]

\section{INTRODUCTION}

The equations ${ }^{1-4}$ for the lattice strains that develop in solids under nonhydrostatic compression in a diamond anvil cell (DAC) have been used extensively in the past to analyze the X-ray diffraction data. Such analyses have yielded valuable information on the strength and elasticity as a function of pressure on a number of solids belonging to cubic, hexagonal, and tetragonal crystal systems (e.g., Refs. 5-11). Studies have shown that while the compressive strength derived from the diffraction data is well constrained, the derived elastic moduli often show significant deviations when compared to those obtained from alternate methods such as Brillouin scattering or extrapolation of the elasticity data obtained from the ultrasonic velocity measurement technique at ambient pressure. Any attempt to improve the reliability of the elastic moduli derived from the diffraction method must consider the effect of three main assumptions made in the derivation of the lattice strain equations. ${ }^{1-4}$ (A1) It is assumed that the diffraction shear modulus is given by the weighted harmonic mean of the diffraction shear moduli under Reuss and Voigt limits with a weight parameter $\alpha$ that lies between 0.5 and 1. (A2) A tensor with only the diagonal components describes adequately ${ }^{12,13}$ the stress state at the center of the sample under nonhydrostatic compression in a DAC. (A3) It is assumed that the lattice strain equations

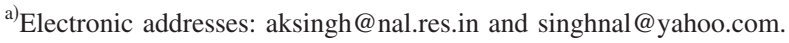

derived using only the linear elasticity theory are adequate to derive compressive strength and elastic moduli from the high-pressure diffraction data. These assumptions have not been put to rigorous scrutiny in the past. In particular, it appears hard to justify A3 in view of the fact that the sample undergoes plastic deformation during nonhydrostatic compression. This has led some investigators ${ }^{14,15}$ to question the adequacy of elasticity based approach.

In this article we examine the validity of these assumptions by considering the published high-pressure diffraction data on four solids of the cubic system: body-centered-cubic (bcc) Fe (Ref. 4 (4.6 GPa), Au (31.9 GPa), Mo (Ref. 5) (21 $\mathrm{GPa}$ ), and $\mathrm{FeO}$ (Ref. 4) (8.3 GPa). The data on Au are unpublished. We used the procedure suggested earlier ${ }^{3,4}$ to derive the strength, single-crystal elastic moduli, and $(h k l)$-dependent diffraction shear moduli in each case. Next, diffraction shear moduli under small deformation are computed using formulae derived by Kröner ${ }^{16}$ and de Wit. ${ }^{17}$ The computed data are subjected to the same analysis ${ }^{3,4}$ to derive the elastic moduli. The results from the analyses of the experimental and computed data are compared to examine the validity of A1. A discussion on the validity of A2 and A3 is presented.

\section{THEORY}

The $d$-spacing measured on the sample under nonhydrostatic compression is given by ${ }^{1-4}$ 


$$
d_{m}(h k l)=d_{P}(h k l)\left[1+\left(1-3 \cos ^{2} \psi\right) Q(h k l)\right] .
$$

$d_{P}(h k l)$ is the $d$-spacing under the mean normal stress $\sigma_{P}$ $=\left(2 \sigma_{1}+\sigma_{3}\right) / 3$. For the cubic system, $d_{P}(h k l)$ is independent of $(h k l) . \psi$ is the angle between the diffraction vector and load axis of the DAC and $Q(h k l)$ is given by

$$
Q(h k l)=t / 6 G^{X}(h k l) .
$$

Here, $t=\left(\sigma_{3}-\sigma_{1}\right)$ and $G^{X}(h k l)$ denotes the diffraction shear modulus for the set of planes $(h k l)$. It is assumed ${ }^{3,4}$ that $G^{X}(h k l)$ is adequately described by the relation

$$
\left[G^{X}(h k l)\right]^{-1}=\left\{\alpha\left[G_{R}^{X}(h k l)\right]^{-1}+(1-\alpha)[G(V)]^{-1}\right\} .
$$

The parameter $\alpha$ determines relative weights of the two types of shear moduli. $G_{R}^{X}(h k l)$ is the diffraction shear modulus calculated under the assumption of stress continuity across the grain boundaries, the averaging being done only over the crystallites that contribute to the diffracted intensity at the point of observation. For the cubic system,

$$
\left[G_{R}^{X}(h k l)\right]^{-1}=2\left[\left(S_{11}-S_{12}\right)-3\left(S_{11}-S_{12}-S_{44} / 2\right) \Gamma(h k l)\right] .
$$

The $S_{i j}$ denote the single-crystal elastic compliances and

$$
\Gamma(h k l)=\left(h^{2} k^{2}+k^{2} l^{2}+l^{2} h^{2}\right) /\left(h^{2}+k^{2}+l^{2}\right)^{2} .
$$

$G(V)$ is the aggregate shear modulus of the polycrystalline solid containing randomly oriented crystallites calculated under the assumption that the strain is continuous across the interfaces between the crystallites. ${ }^{18}$

It may be noted that $d_{P}(h k l)$ and $Q(h k l)$ can be determined from Eq. (1) if $d_{m}(h k l)$ is measured as a function of $\psi$. The experimental technique and method of data analysis are discussed in earlier publications. ${ }^{3,4}$ The parameter $t$ is taken as a measure of the compressive strength of the sample at a pressure $\sigma_{P}$ and can be computed using the relation

$$
t=6 G\langle Q(h k l)\rangle f,
$$

where

$$
\begin{aligned}
& f=A / B, \\
& A=\{[(2 x+3) / 10]+5 x /[2(3 x+2)]\}, \\
& B=\{\alpha[x-3(x-1)\langle\Gamma(h k l)\rangle]+5 x(1-\alpha) /(3 x+2)\}, \\
& x=2\left(S_{11}-S_{12}\right) / S_{44}=2 C_{44} /\left(C_{11}-C_{12}\right) .
\end{aligned}
$$

The symbol \langle\rangle denotes the average over all crystallographic reflections, $C_{i j}$ are the single-crystal elastic moduli, and $G$ is the aggregate shear modulus. It can be easily verified that $f$ $\cong 1$ for $x$ - and $\alpha$-values over a wide range. Experimental $G^{X}(h k l)$ can be obtained from the measured $Q(h k l)$ and $t$ with the help of Eq. (2). Equations (2)-(5) suggest that $Q(h k l)$ versus $3 \Gamma(h k l)$ plot is a straight line with intercept $m_{0}$ and slope $m_{1}$, which are given by ${ }^{4}$

$$
m_{0}=(\alpha t / 3)\left\{S_{11}-S_{12}+\frac{5}{2}\left(\alpha^{-1}-1\right) \frac{\left(S_{11}-S_{12}\right) S_{44}}{3\left(S_{11}-S_{12}\right)+S_{44}}\right\},
$$

$$
m_{1}=-(\alpha t / 3)\left(S_{11}-S_{12}-S_{44} / 2\right) .
$$

The single-crystal elastic compliances and elastic moduli, by inversion of the compliance matrix, can be determined ${ }^{3,4}$ if Eqs. (7a) and (7b) are supplemented with the information on the pressure-dependent compressibility given by

$$
S_{11}+2 S_{12}=1 / 3 K
$$

Kröner ${ }^{16}$ used the concepts put forward by Eshelby ${ }^{19}$ and derived equation for the shear modulus of the isotropic polycrystalline aggregate of crystallites of the cubic system. The Kröner equation of the following form connects aggregate shear modulus $G$ in terms of single-crystal elastic moduli $C_{i j}$,

$$
G^{3}+a G^{2}+b G+c=0 \text {. }
$$

The parameters in Eq. (9) are given by

$$
\begin{aligned}
& a=(3 / 40)\left[15 K+(20 / 3) C^{\prime}\right], \\
& b=-(3 / 8)\left(K C_{44}+4 C^{\prime} C_{44}\right), \\
& c=-(3 / 4) K C^{\prime} C_{44} .
\end{aligned}
$$

Here, $K=\left(C_{11}+2 C_{12}\right) / 3$ and $C^{\prime}=\left(C_{11}-C_{12}\right) / 2$. de $\mathrm{Wit}^{17}$ showed that $G=G^{X}(h k l)$ if the following values of $a, b$, and $c$ are used in Eq. (9),

$$
\begin{aligned}
a= & (3 / 40)\left\{15 K+[12-60 \Gamma(h k l)] C_{44}\right. \\
& \left.-[(16 / 3)-60 \Gamma(h k l)] C^{\prime}\right\}, \\
b= & (3 / 40)\left\{10[1-3 \Gamma(h k l)-(9 / 10)] K C_{44}\right. \\
& \left.+[30 \Gamma(h k l)-6] K C^{\prime}-20 C^{\prime} C_{44}\right\},
\end{aligned}
$$

and

$$
c=-\frac{3}{4} K C_{44} C^{\prime} .
$$

The terms in the original equations of Kröner ${ }^{16}$ and of de $\mathrm{Wit}^{17}$ are rearranged to obtain Eqs. (9), (10a)-(10c), and (11a)-(11c) in compact form. The $G^{X}(h k l)$ at high pressure can be computed by using pressure-dependent $C_{i j}$.

\section{RESULTS}

The details of determining $t$ and $C_{i j}$ from such data are discussed in detail in the earlier articles. ${ }^{3,4,12}$ We use the subscript $m$ to denote the quantities derived from the experimental data. The $Q(h k l)_{m}$ versus $3 \Gamma(h k l)$ plots for $\mathrm{FeO}$, bcc $\mathrm{Fe}$, and Mo are given in the published articles. ${ }^{3,4,12}$ However, these plots together with the plot for $\mathrm{Au}$ are shown in Figs. 1(a) and 1(b) to facilitate discussions given later in this article. The intercept $\left(m_{0}\right)_{m}$ and slope $\left(m_{1}\right)_{m}$ of such plots along with $\left\langle Q(h k l)_{m}\right\rangle$ and $t_{m}$ are listed in Table I.

The $C_{i j}$ values at the relevant pressures were obtained by linear extrapolation using the elastic moduli and their pressure derivatives at ambient pressure measured by ultrasonic velocity technique. The data on $\mathrm{Au}, \mathrm{FeO}$, bcc Fe, and $\mathrm{Mo}$ were taken from Refs. 20-23. It was noted that both linear extrapolation and the formula based on finite strain theory $^{24,25}$ gave nearly identical results. The $C_{i j}$ derived from linear extrapolation are shown in Table II. These extrapolated 


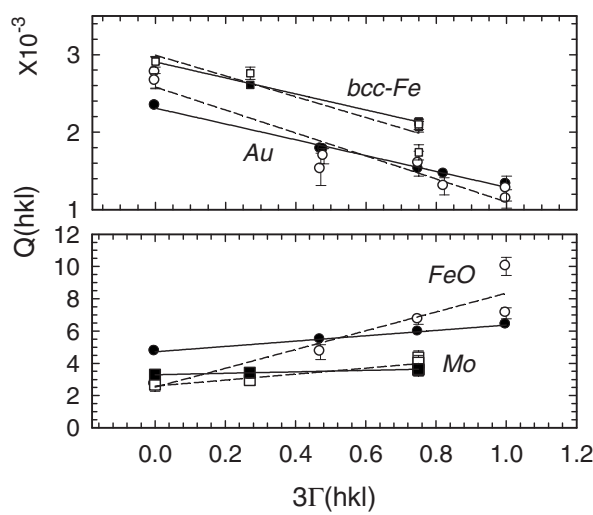

FIG. 1. $Q(h k l)$ vs $3 \Gamma(h k l)$ plots. Unfilled and filled symbols show experimental data and computed data, respectively. Dashed and solid lines are least-squares fits to the experimental and computed data, respectively.

moduli will be used as reference for a comparison with the moduli derived from the diffraction data. The pressuredependent $G$ required for determination of $t$ from Eqs. (6a)-(6e) was obtained from the extrapolated moduli by taking the Voigt-Reuss-Hill (VRH) average. ${ }^{18,26,27}$ The bulk modulus was obtained from the relation $K=\left(C_{11}+2 C_{12}\right) / 3$. The $\left(C_{i j}\right)_{m}$-values were determined from Eqs. (7a), (7b), and (8) for different values of $\alpha$ in the range of $0.4-2$. The $\alpha$-value that gave the $\left(C_{i j}\right)_{m}$-values showing the best overall agreement with the extrapolated data is termed $\alpha_{0}$. These data are given in Table II.

The quantities under small (elastic) deformation are denoted by the suffix "el." The diffraction shear moduli $G^{X}(h k l)_{\mathrm{el}}$ were calculated from Eq. (9). The values of $a, b$, and $c$ were computed using the extrapolated $C_{i j}$ in Eqs. (11a)-(11c). These values are listed in Table III. The $Q(h k l)_{\mathrm{el}^{-}}$-values were calculated from Eq. (2) using the values of $t_{m}$ and $G^{X}(h k l)_{\mathrm{el}}$. The $Q(h k l)_{\mathrm{el}}$ versus $3 \Gamma(h k l)$ plots are shown in Figs. 1(a) and 1(b). The intercepts and slopes of the lines in all four cases are listed in Table I. With these slopes and intercepts, the $\left(C_{i j}\right)_{\mathrm{el}}$-values are computed for different values of $\alpha$. The set of $\left(C_{i j}\right)_{\mathrm{el}}$ that agreed best with extrapolated data and $\alpha_{0}$ is listed in Table II. For a comparison of the values obtained from the VRH average, the aggregate shear

TABLE I. The parameters derived from the plots in Figs. 1(a) and 1(b). Subscripts el and $m$ denote the parameters derived from synthesized and measured data, respectively. $\Delta$ denotes the standard error.

\begin{tabular}{lcccc}
\hline \hline & $\begin{array}{c}\text { bcc Fe } \\
(4.3 \mathrm{GPa})\end{array}$ & $\begin{array}{c}\mathrm{Au} \\
(31.9 \mathrm{GPa})\end{array}$ & $\begin{array}{c}\mathrm{Mo} \\
(21 \mathrm{GPa})\end{array}$ & $\begin{array}{c}\mathrm{FeO} \\
(8.3 \mathrm{GPa})\end{array}$ \\
\hline$\left(m_{0}\right)_{\mathrm{el}}$ & 0.002907 & 0.002310 & 0.003290 & 0.004713 \\
$\left(m_{0}\right)_{m}$ & 0.00299 & 0.00258 & 0.00259 & 0.00253 \\
$\Delta\left(m_{0}\right)_{m}$ & $(0.00016)$ & $(0.00010)$ & $(0.00028)$ & $(0.00071)$ \\
$\left(m_{1}\right)_{\mathrm{el}}$ & -0.001034 & -0.001021 & 0.000454 & 0.001656 \\
$\left(m_{1}\right)_{m}$ & -0.00134 & -0.00148 & 0.00185 & 0.00252 \\
$\Delta\left(m_{1}\right)_{m}$ & $(0.00015)$ & $(0.00015)$ & $(0.00047)$ & $(0.00011)$ \\
$\left\langle Q_{m}(h k l)\right\rangle$ & 0.00234 & 0.00173 & 0.00352 & 0.00560 \\
$G(\mathrm{GPa})$ & $90.90^{\mathrm{a}}$ & $62.44^{\mathrm{a}}$ & $154.67^{\mathrm{a}}$ & $47.03^{\mathrm{a}}$ \\
& $90.2^{\mathrm{b}}$ & $60.5^{\mathrm{b}}$ & $154.4^{\mathrm{b}}$ & $47^{\mathrm{b}}$ \\
$t_{m}(\mathrm{GPa})$ & 1.3 & 0.6 & 3.3 & 1.6 \\
$\Delta t_{m}$ & 0.1 & 0.1 & 0.8 & 0.2 \\
\hline
\end{tabular}

${ }^{\mathrm{a}}$ From Eqs. (9) and (11a)-(11c).

${ }^{\mathrm{b}}$ VRH average.
TABLE II. Comparison of the elastic moduli obtained by extrapolation and analysis of the computed (el) and measured $(m) Q(h k l)$. The numbers inside the square brackets are $\alpha_{0} . \Delta\left(\alpha_{0}\right)$ is standard error in $\alpha_{0}$ and the corresponding errors in $\left(C_{i j}\right)_{m}$ are shown in parentheses.

\begin{tabular}{lcccc}
\hline \hline$C_{i j}$ & bcc Fe $(4.3 \mathrm{GPa})$ & $\mathrm{Au}(31.9 \mathrm{GPa})$ & $\mathrm{Mo}(21 \mathrm{GPa})$ & $\mathrm{FeO}(8.3 \mathrm{GPa})$ \\
\hline$C_{11}$ & 262 & 407 & 599 & 283 \\
$\left(C_{11}\right)_{\mathrm{el}}$ & $261[0.45]$ & $408[0.4]$ & $597[0.5]$ & $285[0.45]$ \\
$\left(C_{11}\right)_{m}$ & $273(7)[0.75]$ & $415(3)[0.75]$ & $594(9)[2.0]$ & $290(7)[1.7]$ \\
$C_{12}$ & 155 & 351 & 235 & 144 \\
$\left(C_{12}\right)_{\mathrm{el}}$ & $155[0.45]$ & $350[0.4]$ & $236[0.5]$ & $143[0.45]$ \\
$\left(C_{12}\right)_{m}$ & $149(3)[0.75]$ & $346(2)[0.75]$ & $238(5)[2.0]$ & $140(5)[1.7]$ \\
$C_{44}$ & 128 & 101 & 138 & 36 \\
$\left(C_{44}\right)_{\mathrm{el}}$ & $129[0.45]$ & $99[0.4]$ & $139[0.5]$ & $36[0.45]$ \\
$\left(C_{44}\right)_{m}$ & $134(6)[0.75]$ & $103(4)[0.75]$ & $137(3)[2.0]$ & $38(2)[1.7]$ \\
$\Delta\left(\alpha_{0}\right)$ & 0.14 & 0.09 & 0.4 & 0.2 \\
\hline \hline
\end{tabular}

modulus was also computed using Eqs. (9) and (10a)-(10c). These values are also shown in Table I. The experimental diffraction shear moduli $\left[G^{X}(h k l)\right]_{m}$ were computed using the $Q(h k l)_{m}$ and $t_{m}$ in Eq. (2) and are listed in Table III.

\section{DISCUSSION}

\section{A. $C_{i j}$ and $t$ from diffraction data}

In all the four cases, it is seen from Fig. 1 and Table I that the slopes of the straight lines drawn through the $Q(h k l)_{m}$ versus $3 \Gamma(h k l)$ data are much larger than the corresponding values for the lines through the $Q(h k l)_{\mathrm{el}}$ versus $3 \Gamma(h k l)$ data. The intercepts of the lines for bcc $\mathrm{Fe}$ and $\mathrm{Au}$ show similar trends. However, the trend for intercepts is reversed in case of $\mathrm{FeO}$ and Mo. In the formulation of the lattice strain theory, the stresses in sample are considered after the flow of the sample between the anvils has ceased. The parameter $t$ is comparable to the yield strength. For this reason, an equivalent of $t_{\mathrm{el}}$ has no significance. The $Q(h k l)_{\mathrm{el}^{-}}$-values were derived from Eq. (2) by using $\left[G^{X}(h k l)\right]_{\mathrm{el}}$ and $t_{m}$. It is readily seen from Eqs. (7a) and (7b) that the value of $t_{m}$ used to derive $Q_{\mathrm{el}}(h k l)$ does not affect the values of $\left(C_{i j}\right)_{\mathrm{el}}$. This was done so that a common procedure could be followed to derive both $\left(C_{i j}\right)_{m}$ and $\left(C_{i j}\right)_{\mathrm{el}}$.

It is convenient to discuss the effect of $\alpha$ on the derived elastic moduli by considering the percentage deviations of $\left(C_{i j}\right)_{m}$ from the extrapolated data given by

$$
\Delta C_{i j}=\left[\left(C_{i j}\right)_{m}-C_{i j}\right] \times 100 / C_{i j} .
$$

Figure 2 shows $\Delta C_{i j}$ as functions of $\alpha$. In all four cases, the deviations of $\Delta C_{11}$ and $\Delta C_{12}$ have nearly equal magnitudes but opposite signs such that at some value of $\alpha$, both become nearly zero. However, $\Delta C_{44}$ does not become zero at this value of $\alpha$. Similar dependencies of $\left(C_{i j}\right)_{m}$ on $\alpha$ have been noticed in an earlier study. ${ }^{9}$ For some value of $\alpha\left(=\alpha_{0}\right)$, the $\Delta C_{11}, \Delta C_{12}$, and $\Delta C_{44}$ are of nearly equal magnitude. The $\left(C_{i j}\right)_{m}$-values obtained with $\alpha_{0}$ were taken as the best match with the extrapolated $C_{i j}$ and are listed in Table II along with the $\alpha_{0}$. It is seen that $\left(C_{i j}\right)_{m}$ for these $\alpha_{0}$-values show good overall match with the extrapolated $C_{i j}$. It was observed that the data sets from different runs on the same material required slightly different $\alpha_{0}$. 
TABLE III. A comparison of diffraction shear moduli computed (subscript el) from Eqs. (9) and (11a)-(11c) from the measured data (subscript $m$ ) and computed from Eq. (3). The $\alpha_{0}$-values used in Eq. (3) are shown in square brackets. Standard errors in $G^{X}(h k l)_{m}$ are shown in parentheses.

\begin{tabular}{|c|c|c|c|c|c|}
\hline$h k l$ & Sample & $\begin{array}{c}G^{X}(h k l)_{\mathrm{el}} \\
\quad(\mathrm{GPa})\end{array}$ & From Eq. (3) & $\begin{array}{c}G^{X}(h k l)_{m} \\
\quad(\mathrm{GPa})\end{array}$ & From Eq. (3) \\
\hline \multirow[t]{2}{*}{110} & bcc Fe & 96.9 & $96.7[0.45]$ & $100(8)$ & $96[0.6]$ \\
\hline & Mo & 151.5 & $151.4[0.5]$ & $126(17)$ & $140[2]$ \\
\hline \multirow[t]{2}{*}{111} & $\mathrm{Au}$ & 78.1 & $80.9[0.4]$ & $91(11)$ & $87[0.6]$ \\
\hline & $\mathrm{FeO}$ & 41.8 & $42.3[0.45]$ & $38(5)$ & $30[1.7]$ \\
\hline \multirow[t]{4}{*}{200} & $\mathrm{Au}$ & 44.2 & $44.2[0.4]$ & $37(4)$ & $37[0.6]$ \\
\hline & $\mathrm{FeO}$ & 56.4 & $56.8[0.45]$ & $99(14)$ & 97 [1.7] \\
\hline & bcc Fe & 70.8 & $71.4[0.45]$ & $71(5)$ & $65[0.6]$ \\
\hline & Mo & 167.1 & $167.9[0.5]$ & $206(32)$ & $220[2]$ \\
\hline \multirow[t]{2}{*}{211} & bcc Fe & 96.9 & $96.7[0.45]$ & $98(8)$ & $96.2[0.6]$ \\
\hline & Mo & 151.5 & $151.4[0.5]$ & $154(21)$ & $140[2]$ \\
\hline \multirow[t]{4}{*}{220} & $\mathrm{Au}$ & 68.0 & $67.0[0.4]$ & $65(8)$ & $65[0.6]$ \\
\hline & $\mathrm{FeO}$ & 45.0 & $45.2[0.45]$ & $40(5)$ & $37[1.7]$ \\
\hline & bcc Fe & 96.9 & $96.7[0.45]$ & $119(11)$ & $96[0.6]$ \\
\hline & Mo & 151.5 & $151.4[0.5]$ & $136(19)$ & $140[2]$ \\
\hline \multirow[t]{2}{*}{310} & bcc Fe & 79.2 & $78.8[0.45]$ & $75(6)$ & $74[0.6]$ \\
\hline & Mo & 161.3 & $161.6[0.5]$ & $186(26)$ & $183[2]$ \\
\hline \multirow[t]{2}{*}{311} & $\mathrm{Au}$ & 58.0 & $56.2[0.4]$ & $68(9)$ & $51[0.6]$ \\
\hline & $\mathrm{FeO}$ & 48.9 & $48.9[0.45]$ & $57(9)$ & $48[1.7]$ \\
\hline \multirow[t]{2}{*}{222} & $\mathrm{Au}$ & 78.1 & $80.9[0.4]$ & $81(11)$ & $87[0.6]$ \\
\hline & $\mathrm{FeO}$ & 41.8 & $42.3[0.45]$ & $27(4)$ & $30[1.7]$ \\
\hline \multirow[t]{2}{*}{400} & $\mathrm{Au}$ & 44.2 & $44.2[0.4]$ & $39(4)$ & $37[0.6]$ \\
\hline & $\mathrm{FeO}$ & 56.4 & $56.8[0.45]$ & $99(15)$ & $97[1.7]$ \\
\hline 331 & $\mathrm{Au}$ & 70.8 & $70.6[0.4]$ & $79(9)$ & $70[0.6]$ \\
\hline 420 & $\mathrm{Au}$ & 58.3 & $56.5[0.4]$ & $61(6)$ & $51[0.6]$ \\
\hline
\end{tabular}

It is seen that the value of $\alpha_{0}$-values for bcc Fe and $\mathrm{Au}$ is 0.75 . These values lie within the range $(0.5<\alpha<1)$ proposed earlier. ${ }^{3,4}$ However, the $\alpha_{0}$-values for $\mathrm{Mo}$ and $\mathrm{FeO}$ are 2 and 1.7, respectively, which are far greater than unity, the upper limit of $\alpha$ used in earlier studies. ${ }^{1-9,12,13}$ It is to be noted that $x>1$ for bcc $\mathrm{Fe}$ and $\mathrm{Au}$, whereas for $\mathrm{Mo}$ and $\mathrm{FeO}$, $x<1$. These results suggest a possible trend that $\alpha_{0}<1$ for

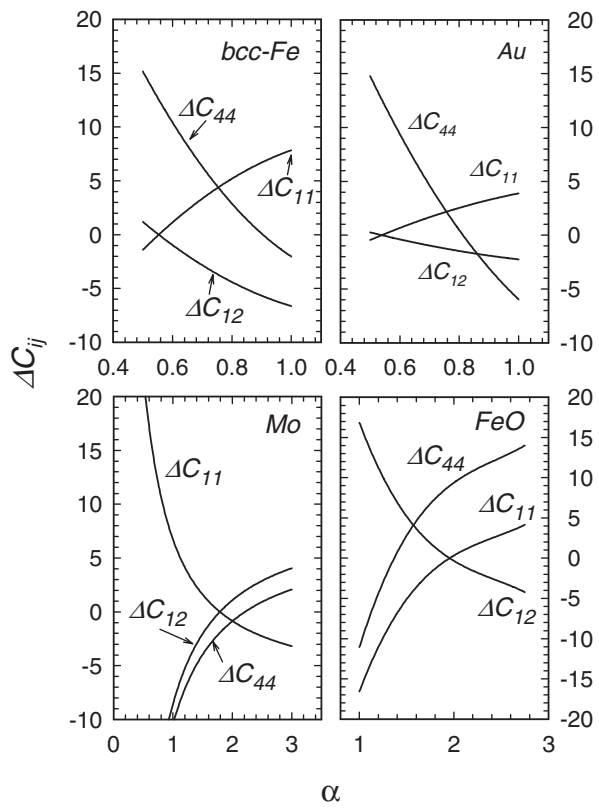

FIG. 2. Percent deviation $\Delta C_{i j}$ of x-ray measured elastic moduli from corresponding extrapolated values as function of $\alpha$. solids with $x>1$ and $\alpha_{0}>1$ for solids with $x<1$. Both $\mathrm{Au}$ and $\mathrm{FeO}$ are face centered cubic but the dependences of $\alpha_{0}$ on $x$ are different. Similarly, Fe and Mo are bcc with different dependencies. Such dependence of $\alpha_{0}$ on $x$ is not related to the crystal structure and slip systems active during deformation. An earlier study ${ }^{28}$ reported $\alpha>1$ for $\mathrm{MgO}$ at modest pressure of 4.7 GPa when plastic deformation was induced by heating the sample. Since $x>1$ for $\mathrm{MgO}$, this contradicts the trend observed in the present study. In the absence of any explanation, studies of this nature on more solids of the cubic system are required to confirm the observed dependence of $\alpha_{0}$ on $x$. The $\left(C_{i j}\right)_{\mathrm{el}}$ derived from $Q_{\mathrm{el}}(h k l)$ are also shown in Table II. It is seen that $\alpha_{0} \cong 0.5$ in all four cases.

The diffraction shear moduli $G^{X}(h k l)_{\mathrm{el}}$ and $G^{X}(h k l)_{m}$ are listed in Table III. It is seen that Eq. (3) with $\alpha_{0} \cong 0.5$ reproduces $G^{X}(h k l)_{\text {el }}$ very well in all four cases. The $\alpha_{0}$ required in Eq. (3) that give the best match of diffraction shear moduli with $G^{X}(h k l)_{m}$ are same as those in Table II for $\mathrm{Mo}$ and $\mathrm{FeO}$ and marginally smaller for bcc $\mathrm{Fe}$ and $\mathrm{Au}$. Since shear moduli are inversely proportional to $Q(h k l)$, the dependences of $G^{X}(h k l)_{\mathrm{el}}$ and $G^{X}(h k l)_{m}$ on $3 \Gamma(h k l)$ are similar to those shown in Fig. 1 with a difference that the slopes of the lines are reversed; it is positive for bcc $\mathrm{Fe}$ and $\mathrm{Au}$ and negative for $\mathrm{Mo}$ and $\mathrm{FeO}$.

The strength $t_{m}$ has been determined in most studies in the past by assuming $f=1$ in Eq. (6a). It can be easily shown from Eqs. (6b)-(6e) that $f$ does not deviate appreciably from unity for $x$-values commonly encountered and $\alpha$-values over a wide range. In this analysis, the $f$-values are $0.83,0.90$, 
1.03, and 0.98 for $\mathrm{Au}$, bcc Fe, Mo, and $\mathrm{FeO}$, respectively. By computing $f$ for various values of $\alpha$, it can be readily seen that for solids with $x>1, f$ deviates from unity more rapidly with increasing anisotropy than for solids with $x<1$. Thus, $t_{m}$ is less sensitive to the uncertainty in the choice of correct $\alpha$ than the elastic moduli.

A few comments on the estimation of standard errors in various derived quantities are in order. The errors $\Delta\left(m_{0}\right)_{m}$ and $\Delta\left(m_{1}\right)_{m}$ are obtained from the straight-line fit to the $Q(h k l)$ versus $3 \Gamma(h k l)$ data. The errors in $\alpha_{0}$ are estimated by carrying out error propagation using Eqs. (7a) and (7b) and $\Delta\left(m_{0}\right)_{m}$ and $\Delta\left(m_{1}\right)_{m}$. The errors in $\left(C_{i j}\right)_{m}$ correspond to the errors in $\alpha_{0}$. The errors in $G^{X}(h k l)_{m}$ and $t_{m}$ are obtained by carrying out error propagation using Eqs. (2) and (6a), respectively, and the errors in $Q(h k l)$.

\section{B. Shear moduli}

A brief discussion of various types of shear moduli used in Sec. II is given in Secs. IV B 1 and IV B 2.

\section{Aggregate shear moduli}

The aggregate shear modulus $G$ is the shear modulus of a polycrystalline solid containing randomly oriented crystallites, which would be obtained by subjecting the solid to a suitable applied stress and measuring the strain by a macroscopic technique. The early attempts to relate $G$ in terms $C_{i j}$ were made with two different assumptions. Voigt ${ }^{18}$ assumed (isostrain or Voigt limit) that the strains across the boundaries separating the crystallites in a polycrystalline aggregate were constant. The shear modulus under this assumption is given by

$$
G(V)=\left(C_{11}-C_{12}+3 C_{44}\right) / 5 .
$$

In an alternate approach, Reuss ${ }^{26}$ assumed (isostress or Reuss limit) that stresses were continuous across the boundaries separating differently oriented crystallites in the polycrystalline solid. Under this assumption, the shear modulus is given by

$$
G(R)=5 C_{44}\left(C_{11}-C_{12}\right) /\left[3\left(C_{11}-C_{12}\right)+4 C_{44}\right] .
$$

Hill $^{27}$ showed that $G(V)$ and $G(R)$ represent the upper and lower bounds, respectively, of the aggregate shear modulus $G$. It is found empirically that $G$ lies close to the average (VRH average) of $G(V)$ and $G(R)$. The difference between the two bounds increases with increasing $x$. For a given value of $x$, the upper and lower bounds proposed by Hashin and Shtrikman ${ }^{29,30}$ constrain $G$ much better than those proposed by Voigt and Reuss. However, in actual cases, the VRH average is found to be close to the average of Hashin and Shtrikman bounds. For this reason, the VRH average is commonly used. As an independent check, $G$ was computed directly from $C_{i j}$ using Eqs. (9) and (10a)-(10c). It can be seen from Table I. that in all the four cases considered here, $G$ obtained from VRH average is close to that from Eqs. (9) and (10a) $-(10 \mathrm{c})$.

\section{Diffraction shear moduli}

The diffraction shear modulus $G^{X}(h k l)$ essentially connects the applied stress in the limit of small deformation on an aggregate of randomly oriented crystallite and X-ray measured strain. The diffraction shear modulus depends on $(h k l)$. The $\mathrm{x}$-ray measured strain is generally different from the macroscopic strain. ${ }^{1}$ The two shear moduli $G$ and $G^{X}(h k l)$ differ to the extent that the macroscopic strain differs from the x-ray measured strain. The difference between the two increases with increasing crystal anisotropy and vanishes if the polycrystalline aggregate contains elastically isotropic crystallites.

The expression for the diffraction shear modulus $G_{R}^{X}(h k l)$ [Eq. (4)] is derived under the Reuss limit and represents an average value. While evaluating this average only those crystallites are considered that contribute to the diffracted intensity at the point of observation. $G(R)$ represents average over all possible orientations of the crystallites. For this reason, $G_{R}^{X}(h k l)$ and $G(R)$ are different even though both are evaluated under Reuss limit. Taking $\Gamma(h k l)$ as a continuous variable not confined only to the crystallographic reflections $(h k l)$, the average value for all possible orientation is given by $\bar{\Gamma}=1 / 5$. It can be easily verified that with $\Gamma(h k l)=\bar{\Gamma}$, Eq. (4) reduces to Eq. (14). The $G(V)$ for the aggregate can be obtained $^{16}$ from Eq. (4) by letting $\Gamma(h k l)=1 /[3+(2 / x)]$.

The expression for the diffraction shear modulus [Eq. (3)] is an extension of an empirical relation that was proposed for the diffraction elastic moduli in the early days of residual stress measurement by x-ray diffraction. A list of these articles can be found in two review articles. ${ }^{31,32}$ The measurements of diffraction elastic constants were usually made by subjecting the test specimen to uniaxial stresses in the elastic range. In such cases, the measured diffraction elastic constants agreed well with those computed from $C_{i j}$ using a relation of the form given by Eq. (3) with $\alpha \approx 0.5 .^{31-34}$ The questions arose about the strict validity of Eq. (3) as $\alpha$ was found to be slightly material dependent in some cases. $^{32}$ Further, Eq. (3) with $\alpha=0.5$ did not fit the experimental data when specimens underwent plastic deformation. An exhaustive list of references on this aspect can be found in Refs. 31 and 32. More recent measurements using neutron diffraction bring to focus the complexity in the applied stress versus measured lattice strain data when the yield stress is approached. ${ }^{35}$ Since Eq. (3) was introduced in the lattice strain theory as an extension of a relation that was considered empirical, ${ }^{31}$ it is important to validate it using $G^{X}(h k l)_{\mathrm{el}}$-data. The data in Table III show that Eq. (3) with $\alpha \approx 0.5$ reproduces the $G^{X}(h k l)_{\mathrm{el}^{-}}$-data very well in all cases. The $\alpha$-values required in the computation of the diffraction shear moduli from Eq. (3) that would match $G^{X}(h k l)_{m}$ are listed in Table III. It is seen that these $\alpha$-values are close to those required for the best match of $\left(C_{i j}\right)_{m}$ with the extrapolated $C_{i j}$.

There are basic differences between the two sets of diffraction shear moduli. $G^{X}(h k l)_{m}$ is derived from experimental data that contain measurement errors as well as the errors due to departure of experimental conditions from the ideal. Most importantly, it contains the effect of plastic deforma- 
tion of the sample. In contrast, being computed data, $G^{X}(h k l)_{\mathrm{el}}$ is free from any experimental errors and the effect of plastic deformation. Two obvious conclusions can be drawn from the data in Table III. The relevant value for $\alpha$ in Eq. (3) is close to 0.5 if the deformation is elastic. The analysis of experimental data that contain the effect of plastic deformation requires $\alpha<1$ if $x>1$ and $\alpha>1$ if $x<1$. These aspects are discussed in Secs. IV C and IV D.

\section{Elastic and plastic strains}

During nonhydrostatic compression in a DAC, the sample-gasket assembly flows radially. The flow ceases when the frictional force at the interfaces between the diamond faces and the gasket-sample assembly balances the stresses causing the flow. In view of such large plastic deformation of the sample, the theory that considers only the elastic strains appears inadequate. ${ }^{14,15}$ However, it should be noted that a diffraction method measures the lattice strains that are elastic. Under high pressures, the lattice strains can be very large. The major fraction of this strain is the volume strain produced by the mean normal stress $\sigma_{P}$; the strain produced by the deviatoric stress component is comparatively small. The volume strains are handled by a suitable equation of state. The linear elasticity theory is applied to derive the expression for the strains produced by only the deviatoric stress. The magnitude of the deviatoric stress is of the order of the yield stress of the sample material. The strains produced by it are not small in absolute sense but the use of linear elasticity theory is still justified in view of rather large errors (as compared to those in macroscopic strain-measurement techniques that are used at ambient pressures) in the measurement of lattice strains. The point often overlooked is that only the x-ray measured strains are used in the entire analysis of the high-pressure x-ray diffraction data. These strains are elastic. The stresses such as $\sigma_{P}$ and $t$ are computed from the measured lattice strains using appropriate elastic moduli. The applied stress does not form an input in the entire analysis. For this reason, the anomalies in the applied stress versus strain data observed in many ambientpressure studies ${ }^{31,32,35}$ and discussed briefly in the last paragraph of Sec. IV B 2 do not directly translate to the present case even though the measurements are made on severely deformed samples. This probably would explain why the theory, despite its many seeming drawbacks, is successful in yielding $\sigma_{P}, t$ with high certainty and $C_{i j}$ with comparatively lower certainty.

The texturing (preferred orientation) is an inevitable consequence of plastic deformation of a polycrystalline solid. The derivation of the expression for diffraction shear modulus [Eq. (4)] requires the averages of trigonometric terms containing a parameter $\varphi$, an angle that defines the orientation of the reflecting planes about reflecting-plane normal ( $\varphi$-group of crystallites). ${ }^{1}$ The lattice strain equations are derived assuming a random distribution of the crystallites such that the number of crystallites with orientation $n(\varphi)$ is independent of $\varphi$. This gives $\langle\cos \varphi\rangle=\langle\sin \varphi\rangle=0$ and $\left\langle\cos ^{2} \varphi\right\rangle=\left\langle\sin ^{2} \varphi\right\rangle=1 / 2$. The presence of texture alters these averages. It can be seen that the averages for a distribution $n(\varphi)=n(-\varphi)$ are the same as those for a random distribution. Therefore, only the component of the distribution that deviates from the distribution with a center of inversion will affect the averages of the required trigonometric terms.

The parameter $t$ represents the yield strength of the bulk polycrystalline sample. At any angle $\psi$, the plane normal of the diffracting planes of the $\varphi$-group of crystallites has a definite orientation with respect to the load axis. This orientation changes with the changing $\psi$-angle. In analogy with the yield behavior of single crystals, it is reasonable to expect orientation-dependent yielding of the crystallites constituting the polycrystalline aggregate. In such a case, one may expect $(h k l)$-dependent $t$. A simple model based on the concepts of single-crystal yielding ignoring the constraint imposed by the neighboring crystallites on the deformation of an individual crystallite was developed. ${ }^{36}$ The results of this model, however, did not fit the $d_{m}(h k l)$ versus $\psi$ data.

In a recent publication, Karato ${ }^{37}$ extended the theory of lattice strains in a polycrystalline aggregate to include the effect of plastic deformation. However, usefulness of this theory in the interpretation of high-pressure diffraction patterns will be known in due course of time.

\section{Bounds for diffraction shear modulus}

In earlier high-pressure $\mathrm{X}$-ray diffraction studies, ${ }^{28,38}$ cases of $\alpha>1$ were observed after the onset of plastic deformation of the sample. These authors concluded that the Reuss and Voigt limits do not bound the elastic moduli when large plastic strain accumulates in the sample. This conclusion implied the extension of Hill's analysis ${ }^{27}$ to the diffraction shear moduli. It should be emphasized that the Hill's analysis is strictly applicable to the elastic moduli of polycrystalline aggregates of randomly oriented crystallites. It is not necessarily valid for the diffraction shear moduli, as $G^{X}(h k l)$ is distinctly different from the aggregate shear modulus $G$. It is possible to check this by comparing $G^{X}(h k l)_{\mathrm{el}}$ with $G_{R}^{X}(h k l)$ and $G(V)$. Figure 3 shows this comparison for the four cases. Many instances of $G_{R}^{X}(h k l)$ $>G(V)$ are seen. This suggests that the shear modulus under Voigt limit does not always constitute the upper bound as it does in the case of aggregate shear modulus. ${ }^{27}$ It can be shown using Eqs. (3) and (13) that $G_{R}^{X}(h k l)>G(V)$ for a reflection if $\Gamma(h k l)>1 /(3+2 / x)$ for $x>1$ and $\Gamma(h k l)$ $<1 /(3+2 / x)$ for $x<1$. Further, $G_{R}^{X}(h k l)<G(R)$ if $\Gamma(h k l)$ $<1 / 5$ for $x>1$ and $\Gamma(h k l)>1 / 5$ for $x<1$. However, for all reflections $G^{X}(h k l)_{\mathrm{el}}$ lies nearly halfway between $G_{R}^{X}(h k l)$ and $G(V)$. This explains why Eq. (3) with $\alpha \cong 0.5$ gives $G^{X}(h k l)_{\mathrm{el}}$ correctly. Thus, diffraction shear modulus under Reuss and Voigt limits does not give the lower and upper bounds of the diffraction shear modulus as the shear moduli under Reuss and Voigt limits do for the aggregate shear modulus. $^{27}$

Clearly, cases of $\alpha>1$ arise due to the effect of plastic deformation of the sample on the measurements of $G^{X}(h k l)$. However, the present analysis gives $\alpha>1$ for only Mo and $\mathrm{FeO}$. Although the samples of $\mathrm{Au}$ and $\mathrm{Fe}$ also undergo plastic deformation, the analysis suggests $\alpha<1$. As pointed out earlier in this article, $\alpha>1$ for solids with $x<1$ and $\alpha<1$ for 


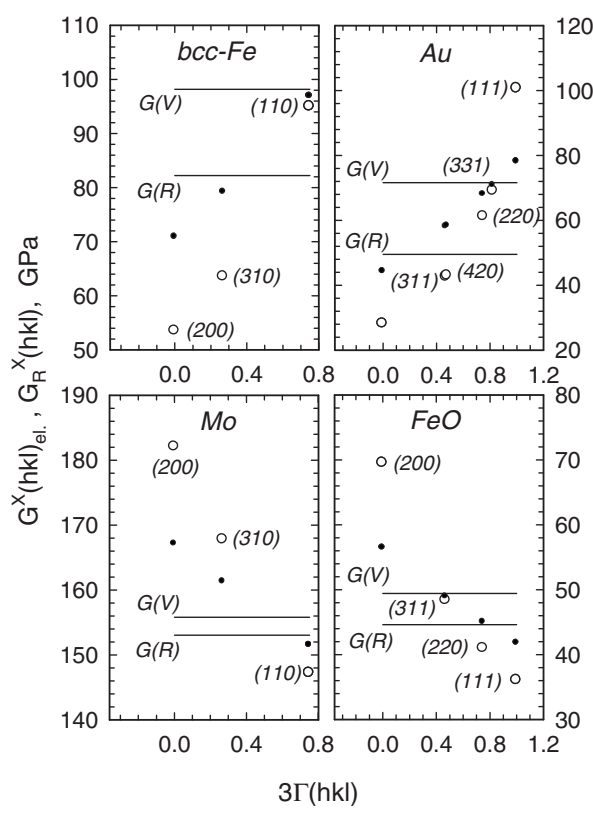

FIG. 3. $G^{X}(h k l)_{\text {el }}$ computed from Eqs. (9) and (11a)-(11c) (filled circles) and $G_{R}^{X}(h k l)$ computed from Eq. (4) (unfilled circles) as a function of $3 \Gamma(h k l)$. Unfilled circle (marked with $h k l$ ) and corresponding filled circle are located at the same $3 \Gamma(h k l)$ value.

those with $x>1$. This indicates that plastic deformation of the sample does not directly affect the measurements. A possible way the plastic deformation can affect the diffraction data is discussed below.

The plastic deformation of the sample compressed between the anvils gives rise radial stress gradients. In an ideal setup, the diamond faces must remain parallel under load such that the maximum pressure (stress) occurs at the geometric center of the sample. The radial stress gradient is zero in a small region at the peak stress. A fine incident beam should illuminate only this region and thereby avoid the diffraction from the region of stress gradient. It is extremely difficult to achieve this experimentally. More difficult to achieve is the condition that the incident beam should not wander as the position of the DAC is rotated to set different $\psi$-values. ${ }^{39}$ Any departure from these ideal conditions causes incident $\mathrm{x}$-ray beam to illuminate the region of appreciable stress gradient. In such a case, the off-diagonal terms appear in the stress tensor and assumption A2 is no longer valid. The diffraction data obtained under these conditions are not strictly amenable to analysis with the present form of lattice strain equations. ${ }^{1-4}$ It is suggested that the observed dependence of $\alpha$ on $x$ arises when assumption A2 is violated. Efforts to minimize experimentally the diffracted intensity from the region of large stress gradients will greatly reduce the uncertainties in the derived parameters.

\section{SUMMARY}

The analysis of radial x-ray diffraction data on four polycrystalline solids of the cubic system suggests that singlecrystal elastic moduli can be derived with a degree of reliability by suitably choosing the value of weighting factor $\alpha\left(=\alpha_{0}\right)$. It is found that $\alpha_{0}<1$ for solids with $x>1$ and $\alpha_{0}$ $>1$ if $x<1$. This trend, however, is empirical and requires further validation. It is suggested that the observed dependence of $\alpha_{0}$ on $x$ arises due to the appearance of off-diagonal terms in the stress tensors when the incident $\mathrm{x}$-ray beam passes through region stress gradients in the sample. Practical application of this observation in deriving single-crystal elastic moduli is limited because $\alpha_{0}$ is not known a priori. Even in the limit of small deformation, the diffraction shear modulus Voigt limit does not give the upper bound of the diffraction shear modulus for all values of $(h k l)$. The diffraction shear modulus, however, lies halfway between the diffraction shear moduli under Reuss and Voigt limits and, thus, giving $\alpha \cong 0.5$ for all $(h k l)$. It is suggested that the uncertainties in the derived parameters can be greatly reduced if diffraction from the regions of stress gradients can be avoided in the experiments.

${ }^{1}$ A. K. Singh, J. Appl. Phys. 73, 4278 (1993); 74, 5920(E) (1993).

${ }^{2}$ T. Uchida, N. Funamori, and T. Yagi, J. Appl. Phys. 80, 739 (1996).

${ }^{3}$ A. K. Singh, H. K. Mao, J. Shu, and R. J. Hemley, Phys. Rev. Lett. 80, 2157 (1998).

${ }^{4}$ A. K. Singh, C. Balasingh, H. K. Mao, R. J. Hemley, and J. Shu, J. Appl. Phys. 83, 7567 (1998).

${ }^{5}$ T. S. Duffy, G. Shen, J. Shu, H. K. Mao, R. J. Hemley, and A. K. Singh, J. Appl. Phys. 86, 6729 (1999).

${ }^{6}$ S. Speziale, S. R. Shieh, and T. S. Duffy, J. Geophys. Res., [Solid Earth] 111, B02203 (2006).

${ }^{7}$ A. K. Singh, H. P. Liermann, S. K. Saxena, H. K. Mao, and S. Usha Devi, J. Phys.: Condens. Matter 18, S969 (2006).

${ }^{8}$ H. K. Mao, J. F. Shu, G. Shen, R. J. Hemley, B. Li, and A. K. Singh, Nature (London) 396, 741 (1998).

${ }^{9}$ T. S. Duffy, G. Shen, D. L. Heinz, J. Shu, Y. Ma, H. K. Mao, R. J. Hemley, and A. K. Singh, Phys. Rev. B 60, 15063 (1999).

${ }^{10}$ S. Merkel, J. Shu, P. Gillet, H. K. Mao, and R. J. Hemley, J. Geophys. Res., [Solid Earth] 110, B05201 (2005).

${ }^{11}$ S. R. Shieh, T. S. Duffy, and B. Li, Phys. Rev. Lett. 89, 255507 (2002).

${ }^{12}$ A. K. Singh and G. C. Kennedy, J. Appl. Phys. 45, 4686 (1974).

${ }^{13}$ A. L. Ruoff, J. Appl. Phys. 46, 1389 (1975).

${ }^{14}$ D. J. Weidner, L. Li, M. Davis, and J. Chen, Geophys. Res. Lett. 31, L06621 (2004).

${ }^{15}$ S. Merkel, J. Phys.: Condens. Matter 18, S949 (2006).

${ }^{16}$ E. Kröner, Z. Phys. 151, 504 (1958).

${ }^{17}$ R. de Wit, J. Appl. Crystallogr. 30, 510 (1997).

${ }^{18}$ W. Voigt, Lehrbuch der Kristallphysik (Teubner, Leipzig, 1928).

${ }^{19}$ J. D. Eshelby, Proc. R. Soc. London, Ser. A 241, 376 (1957).

${ }^{20}$ B. Golding, S. C. Moss, and B. L. Averbach, Phys. Rev. 158, 637 (1967).

${ }^{21}$ I. Jackson, S. K. Khanna, A. Revcolevsci, and J. Berthon, J. Geophys. Res. 95, 671 (1990).

${ }^{22}$ M. W. Guinan and D. N. Besher, J. Phys. Chem. Solids 29, 541 (1968).

${ }^{23}$ K. W. Katahara, M. H. Manghnani, and E. S. Fisher, J. Phys. F: Met. Phys. 9, 773 (1979).

${ }^{24}$ F. Birch, J. Geophys. Res. 83, 1257 (1978).

${ }^{25}$ A. K. Singh, H. P. Liermann, Y. Akahama, S. K. Saxena, and E. Menéndez-Proupin, J. Appl. Phys. 103, 063524 (2008).

${ }^{26}$ A. Reuss, Z. Angew. Math. Mech. 9, 49 (1929).

${ }^{27}$ R. Hill, Proc. Phys. Soc., London, Sect. A 65, 349 (1952).

${ }^{28}$ J. Chen, L. Li, T. Yu, H. Long, L. Wang, D. Weidner, and M. Vaughan, J. Phys.: Condens. Matter 18, S1049 (2006).

${ }^{29}$ Z. Hashin and S. Shtrikman, J. Mech. Phys. Solids 10, 335 (1962).

${ }^{30}$ Z. Hashin and S. Shtrikman, J. Mech. Phys. Solids 10, 343 (1962).

${ }^{31}$ G. B. Greenough, in Progress in Metal Physics, edited by B. Chalmers (Pergamon, New York, 1952), pp. 176-219.

${ }^{32}$ E. Macherauch, Exp. Mech. 6(3), 140 (1966). 
${ }^{33}$ A. K. Singh and C. Balasingh, J. Appl. Phys. 90, 2296 (2001).

${ }^{34}$ T. Gnäupel-Herold, P. C. Brand, and H. J. Prask, J. Appl. Crystallogr. 31 929 (1998).

${ }^{35}$ B. Clausen, T. Lorentzen, M. A. M. Bourke, and M. R. Daymond, Mater. Sci. Eng., A 259, 17 (1999).

${ }^{36}$ A. K. Singh, in Science and Technology of High Pressure-Proceedings of
AIRAPT-17, edited by M. H. Manghnani, W. J. Nellis, and M. F. Nichol (Universities Press India Ltd., Hyderabad, India, 2000), pp. 62-67.

${ }^{37}$ S. Karato, Phys. Rev. B 79, 214106 (2009).

${ }^{38}$ J. Chen, L. Li, D. Weidner, and M. Vaughan, Phys. Earth Planet. Inter. 143-144, 347 (2004).

${ }^{39}$ H. K. Mao and R. J. Hemley, High Press. Res. 14, 257 (1996). 